\title{
How "healthy" is circuit resistance training following paraplegia? Kinematic analysis associated with shoulder mechanical impingement risk
}

\author{
Linda M. Riek, DPT, PhD; ${ }^{*}$ Paula M. Ludewig, PT, PhD; ${ }^{2}$ Deborah A. Nawoczenski, PT, PhD ${ }^{3}$ \\ ${ }^{1}$ Department of Physical Therapy, Nazareth College, Rochester, NY; ${ }^{2}$ Department of Physical Medicine and Rehabilita- \\ tion, Program in Physical Therapy, University of Minnesota, Minneapolis, MN; ${ }^{3}$ Department of Physical Therapy, Ithaca \\ College, Rochester, NY
}

\begin{abstract}
The purpose of the study was to determine whether wheelchair-based circuit resistance training (CRT) exercises place the shoulder at risk for mechanical impingement. Using a novel approach, we created a mechanical impingement risk score for each exercise by combining scapular and glenohumeral kinematic and exposure data. In a case series design, 18 individuals (25-76 yr old) with paraplegia and without substantial shoulder pain participated. The mean mechanical impingement risk scores at 45-60 degrees humerothoracic elevation were rank-ordered from lowest to highest risk as per subacromial mechanical impingement risk: overhead press $(0.6+/-0.5$ points), lat pulldown (1.2 +/- 0.5 points), chest press $(2.4+/-$ 2.8 points), row ( $2.7+/-1.6$ points), and rickshaw $(3.4+/-2.3$ points). The mean mechanical impingement risk scores at 105120 degrees humerothoracic elevation were rank-ordered from lowest to highest risk as per internal mechanical impingement risk: lat pulldown (1.2 +/- 0.5 points) and overhead press (1.3+/- 0.5 points). In conclusion, mechanical impingement risk scores provided a mechanism to capture risk associated with CRT. The rickshaw had the highest subacromial mechanical risk, whereas the overhead press and lat pulldown had the highest internal mechanical impingement risk. The rickshaw was highlighted as the most concerning exercise because it had the greatest combination of magnitude and exposure corresponding with increased subacromial mechanical impingement risk.
\end{abstract}

Key words: biomechanics, circuit resistance training, conditioning, exercise, impingement, kinematics, paraplegia, shoulder, spinal cord injury, wheelchair.

\section{INTRODUCTION}

Following spinal cord injury (SCI), survivors encounter secondary complications associated with a relatively sedentary lifestyle. Exercise is recommended to control high rates of obesity (53\%-66\% are overweight and 20\%$30 \%$ are obese) [1-3], diabetes mellitus (20\%) [3-4], and cardiovascular disease (22\% have high blood pressure) [2$3]$. Because of the nature of SCI, many individuals are limited to upper-limb (UL) exercises as their primary means of conditioning. Studies showed combined ergometry and multistation resistance training improved strength, ergometry performance, anaerobic power, cardiovascular endurance, psychological well-being, oxygen consumption $\left(\mathrm{VO}_{2}\right)$ peak, and atherogenic lipid profiles [5-8]. UL circuit resistance training (CRT) in isolation improved $\mathrm{VO}_{2}$ peak, UL peak and mean power, and UL isotonic strength as measured on CRT exercises that include horizontal

\footnotetext{
Abbreviations: 1-RM = one-repetition maximum, AIS = American Spinal Injury Association Impairment Scale, CRT = circuit resistance training, $\mathrm{SCI}=$ spinal cord injury, $\mathrm{T}=$ thoracic, $\mathrm{UL}=$ upper limb, $\mathrm{VO}_{2}=$ oxygen consumption.

*Address all correspondence to Linda M. Riek, DPT, PhD; Nazareth College, 4245 East Ave, Rochester, NY 14618; 585-389-2132; fax: 585-389-2908. Email: lriek9@naz.edu http://dx.doi.org/10.1682/JRRD.2012.06.0112
} 
press, horizontal row, overhead press, overhead pull, seated dips, and arm curls [9]. For many individuals, CRT is an attractive choice for UL exercise because it is available in home gyms and rehabilitation and community fitness centers and can be performed in a seated position from the wheelchair (Figure 1).
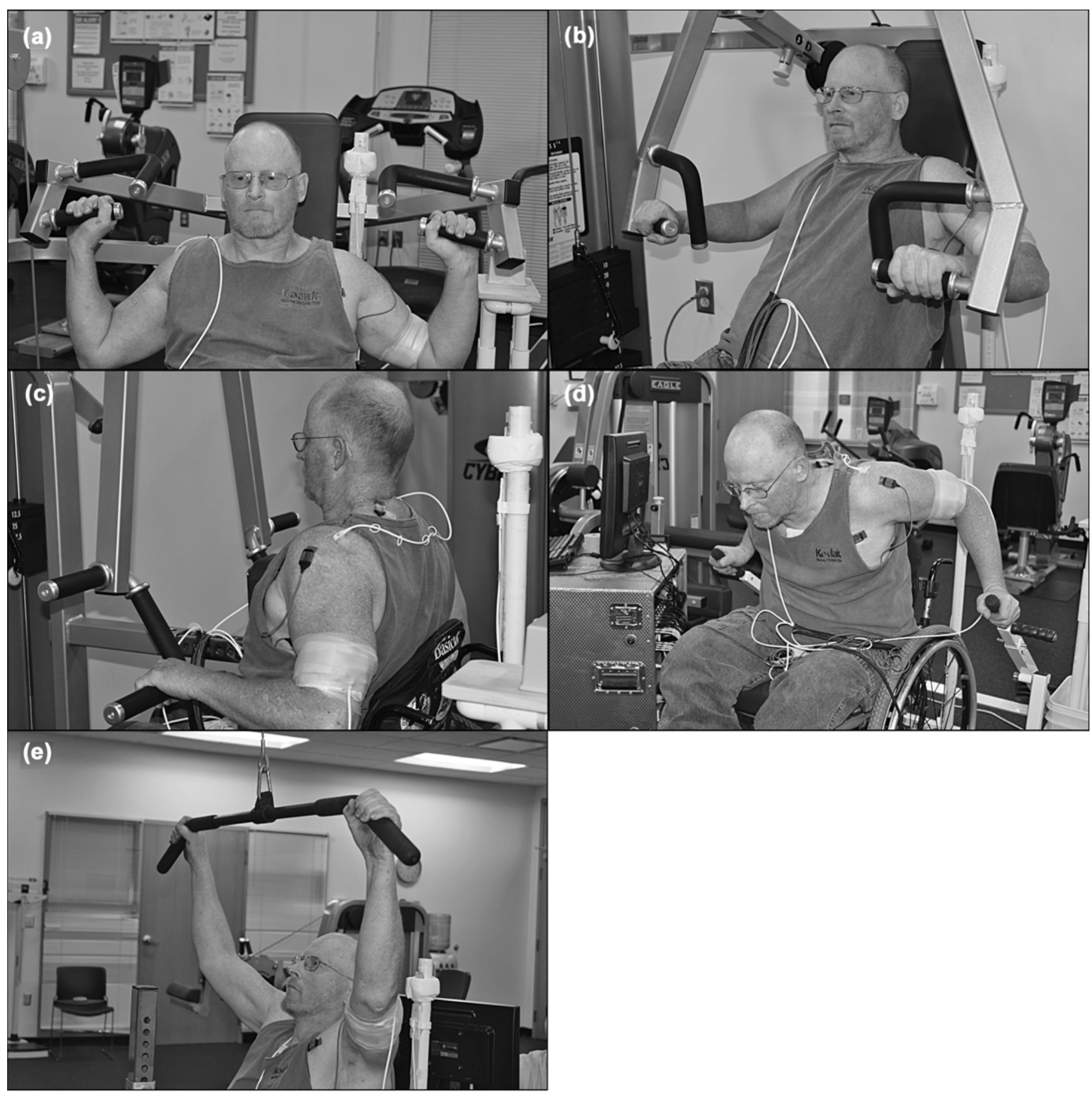

Figure 1.

Circuit resistance training exercises. (a) Overhead press, (b) chest press, (c) seated row, (d) rickshaw, and (e) lat pulldown. 
The general health benefits of CRT exercises are widely recognized. However, given that shoulder pain is also a significant problem in the SCI population, prevention of shoulder impingement and maintenance of shoulder health while performing CRT are of utmost importance. Specifically, 60 to 83 percent of individuals with paraplegia described shoulder pain since beginning wheelchair use [10-11] and between 40 and 67 percent reported current shoulder pain [11-13]. Of those with shoulder pain, over 70 percent are diagnosed with shoulder impingement [14-16].

Shoulder mechanical impingement can be differentiated into subacromial and internal impingement [17-22]. Subacromial impingement involves compression or mechanical irritation of the subacromial bursa, supraspinatus tendon, infraspinatus tendon, and/or long head of the biceps tendon between the coracoacromial arch and the humeral head (Figure 2(a)) [22] and is purported to occur at lower humeral elevation angles. At angles less than $60^{\circ}$ humerothoracic elevation, the greater tuberosity of the humerus approximates the acromion and the supraspinatus tendon is most susceptible to impingement [23]. As the arm elevates beyond $60^{\circ}$, the rotator cuff tendons clear the coracoacromial arch [23-24].
Internal impingement, on the other hand, results from compression or mechanical irritation of the supraspinatus tendon, infraspinatus tendon, and joint capsule between the glenoid and the humeral head. This compression and irritation results in possible articular-sided partial-thickness tears of the supraspinatus or infraspinatus and either posteriorsuperior, posterior, or anterior-superior labral fraying or tears (Figure 2(b)) [20-21,25]. Posterior internal impingement was first described with repetitive extension, abduction, and external rotation of the humerus as seen during throwing and overhead activities, resulting in posterior shoulder pain [25]. Alternatively, anterior internal impingement likely occurs with repetitive flexion, abduction, and internal rotation of the humerus as seen during many overhead activities, resulting in anterior shoulder pain. Internal impingement occurs at higher degrees of humeral elevation (greater than $105^{\circ}$ ) than does subacromial impingement.

CRT exercises need to be prescribed and performed thoughtfully with regard to healthy shoulder biomechanics that minimize positions of impingement. Currently, the potential health benefit of CRT programs as compared with the risk of shoulder impingement during CRT remains unknown, because shoulder biomechanics during CRT have not been investigated. CRT is used under the premise

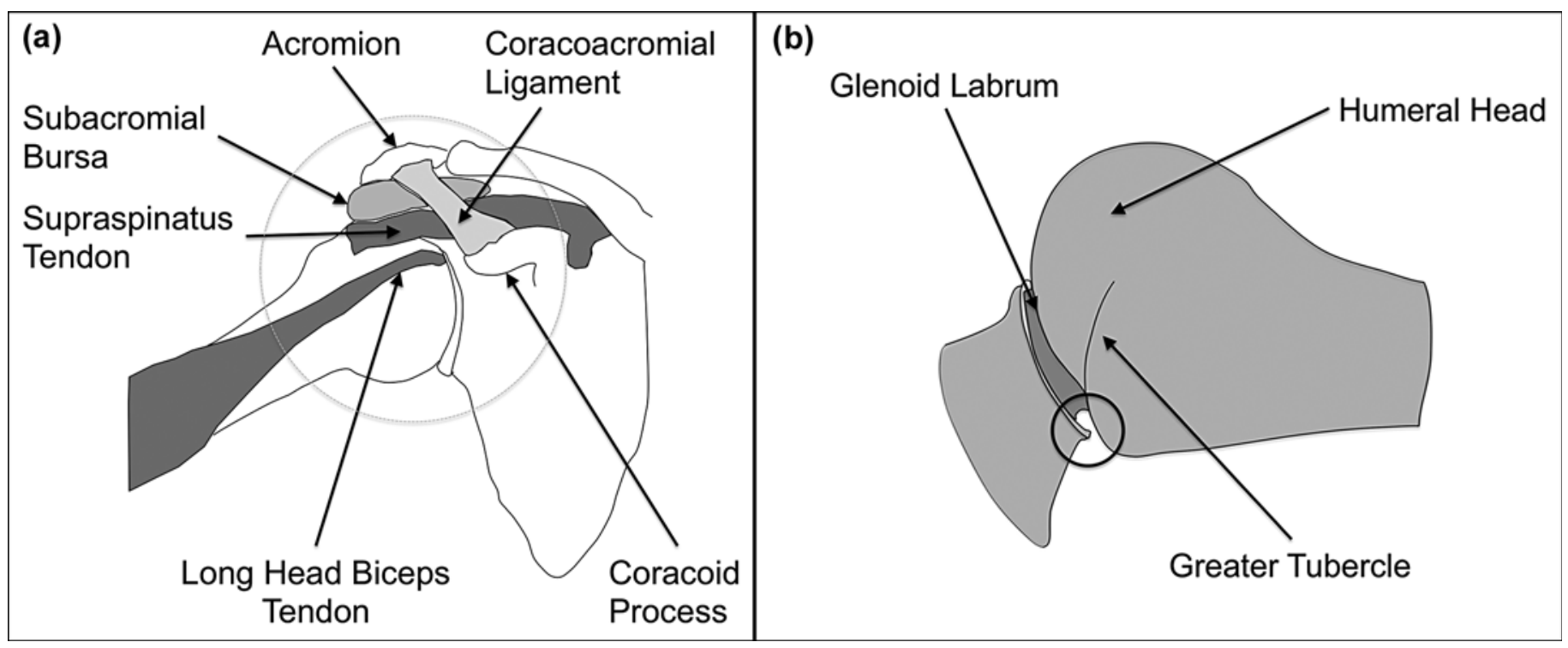

Figure 2.

Impingement. (a) Subacromial/external impingement. Anterior view of right shoulder. Circle indicates area of subacromial/external impingement beneath coracoacromial arch, which is formed by coracoid process, acromion, and coracoacromial ligament. (b) Internal impingement. Posterior view of right shoulder with acromion removed and humerus elevated. Circle indicates area of internal impingement. Joint capsule is not depicted because it encircles entire glenohumeral joint from glenoid cavity to anatomical neck of humerus. 
that it is "healthy." Yet there is no biomechanical evidence that CRT is healthy for the shoulders. In fact, CRT may not be healthy for the shoulder if detrimental scapular and glenohumeral kinematics predominate during the exercises (Figure 3). Certain kinematic positions (decreased scapular posterior tilt [26-28] and decreased upward rotation [26-27,29] have been linked to impingement (Figure 3(a), $x$ - and $y$-axis, respectively). Increased scapular internal rotation during loaded, non-weight-bearing conditions have also been reported for individuals with impingement (Figure 3(a), z-axis) [27,30]. Although increased glenohumeral internal rotation has not been found in individuals with impingement, current literature supports glenohumeral external rotation during humerothoracic elevation as a normal movement pattern in asymptomatic individuals [31]. A reduction in humeral external rotation has been shown to increase subacromial rotator cuff contact (Figure 3(b), z-axis) [32]. Additionally, Yanai et al. demonstrated greater impingement force on the coracoacromial ligament in $90^{\circ}$ abduction plus maximum internal rotation as compared with neutral rotation or external rotation [33]. In summary, using currently available information, decreased scapular posterior tilt and upward rotation and increased scapular and glenohumeral internal rotation are viewed as potentially detrimental kinematics. Of note, the overall kinematics are a result of the balance of forces for any individual, including active and passive muscle forces, as well as ligament and capsular forces at higher angles of elevation.

Although there have been a limited number of exercise programs that focused on treatment of shoulder pain in SCI after it occurs [34-35], there are no studies to date that have investigated shoulder kinematics during the execution of CRT with the intent to reduce mechanical impingement risk. Taking into consideration both the magnitude of combined scapular and glenohumeral kinematics, as well as the exposure (time spent in impingement ranges) during the execution of CRT, may help to guide exercise prescription and thus minimize the risk of shoulder pain associated with impingement.

The purpose of this study was to compare both scapular and glenohumeral kinematics and exposure during wheelchair-based UL CRT exercises at specific humerothoracic elevation angles to determine whether the CRT

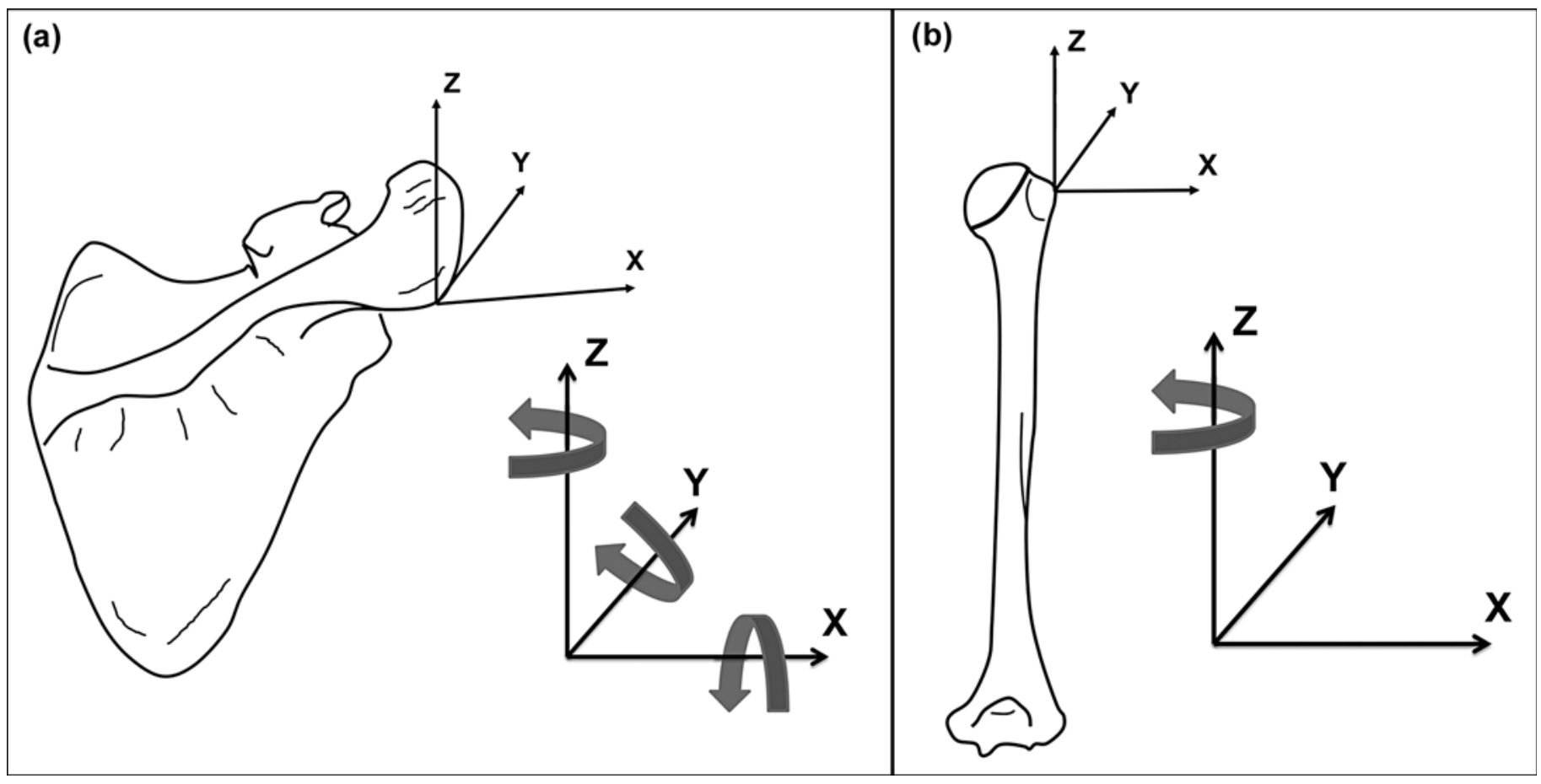

Figure 3.

Scapular and glenohumeral kinematics. Posterior view of (a) right scapula and (b) humerus. Potentially detrimental directions for scapular and glenohumeral kinematic rotations highlighted with arrow. 
exercises place the shoulder at mechanical impingement risk. Subacromial mechanical impingement risk was identified at lower ranges of humerothoracic elevation $\left(45^{\circ}-60^{\circ}\right)$ and internal mechanical impingement risk at upper ranges $\left(105^{\circ}-120^{\circ}\right)$. Based on results from pilot testing, we hypothesized that CRT exercises could be rankordered for subacromial, as well as internal, mechanical impingement risk. CRT exercises will be rank-ordered from lowest-to-highest subacromial mechanical impingement risk as follows: chest press, lat pulldown, seated row, and rickshaw. CRT exercises will be rank-ordered from lowest-to-highest internal mechanical impingement risk as follows: lat pulldown followed by overhead press. The overall goal of this study was to provide CRT exercise recommendations that emphasize healthy shoulder motions to both healthcare practitioners and consumers.

\section{METHODS}

\section{Subjects and Exercise Protocol}

Using a case-series design, we enrolled 20 subjects (15 men and 5 women) ranging in age between 25 and 76 yr with paraplegia from SCI in this study. One male and one female were dropped because of kinematic measurement error, leaving 18 subjects for analysis. American Spinal Injury Association Impairment Scale (AIS) motor levels ranged from thoracic $(\mathrm{T}) 3$ to lumbar 2, and subjects were without shoulder pain as demonstrated by the Wheelchair User's Shoulder Pain Index scores (Table 1). Subjects were recruited from the community as a sample of convenience and were seasoned wheelchair users (2-28 yr). Activity level was representative of previous literature $[34,36]$ with a mean 18 transfers per day. Inclusion criteria were participants at least $18 \mathrm{yr}$ old and $1 \mathrm{yr}$ status post-SCI from trauma, vascular, or orthopedic origin resulting in

Table 1.

Subject demographics.

\begin{tabular}{lcc}
\hline \multicolumn{1}{c}{ Demographic } & $\begin{array}{c}\text { Mean } \pm \text { Standard } \\
\text { Deviation }\end{array}$ & Range \\
\hline Age (yr) & $46.7 \pm 11.4$ & $25-76$ \\
BMI & $26.1 \pm 4.1$ & $18.7-36.0$ \\
Years Post-SCI & $15.7 \pm 8.5$ & $2-28$ \\
WUSPI Score (0-150) & $1.5 \pm 2.7$ & $0-8.4$ \\
Transfers/Day & $18.0 \pm 13.9$ & $4-70$ \\
BMI = body mass index, SCI = spinal cord injury, WUSPI = Wheelchair \\
User's Shoulder Pain Index.
\end{tabular}

paraplegia at AIS T2 motor or below requiring the use of a manual wheelchair for primary mobility. Exclusion criteria included trauma, dislocation, or surgery to the glenohumeral or acromioclavicular joints because this could alter shoulder kinematics. Additionally, participants were excluded if they had self-reported pain beyond a nominal level because pain can potentially alter shoulder kinematics. Shoulder pain was determined by a positive painful arc, self-reported shoulder pain exceeding 3 (maximum score of 10) when determining CRT resistance levels or 10 (maximum score of 150) on the Wheelchair Users Shoulder Pain Index [37].

Participants attended two sessions at a community fitness center equipped with Cybex Total Access equipment (Medway, Massachusetts). This equipment allows participants to remain in their custom wheelchairs while exercising. At the initial session, the Mayhew regression equation was used to safely predict the one-repetition maximum (1-RM) for all five CRT exercises while performing submaximal lifting (Figure 1) [5]. At the second session, scapular and glenohumeral kinematics during CRT were acquired. Subjects completed one set of 10 repetitions for each exercise at 50 percent 1-RM. We chose 50 percent 1-RM based upon previous SCI literature [5-6,38]. Each CRT exercise was completed with a $6 \mathrm{~s}$ pattern paced with a metronome. A minimum 5 min rest was allowed between exercises. To simulate a "real-world" experience, subjects remained in their custom wheelchair and exercises were tested in random order to minimize systematic effects of fatigue or learning.

\section{Data Collection}

Three-dimensional position and orientation were captured by the Flock of Birds electromagnetic tracking system (mini-BIRD model 800, Ascension Technology Corporation; Milton, Vermont). The system to sensor range is $76.2 \mathrm{~cm}$ in any direction with a root-mean-squared accuracy of $1.8 \mathrm{~mm}$ for position and $0.5^{\circ}$ for orientation. This system has been used successfully with previous shoulder investigations in this laboratory [36,39]. Electromagnetic surface markers were adhered to the skin overlying the manubrium and the superior surface of the acromion and to a cuff at the distal humerus. Validity of the surface markers [40] and reliability (intraclass correlation values from 0.83 to 0.99) of the electromagnetic system [29] have been established in previous investigations. Specifically, surface markers and invasive bone pins are deemed comparable with the exception of skin motion artifact for surface markers, which is most consequential when exceeding $120^{\circ}$ shoulder elevation [40]. Prior to data collection, sensors 
were tested at the fitness center to identify whether there was interference in that environment. Translational distortion was less than $2 \mathrm{~mm}$. The left UL was assessed for all subjects because of easier accessibility to CRT equipment.

Bony anatomical points on the thorax, scapula, and humerus were digitized following modified International Society of Biomechanics standards [36,39,41]. For the scapula, the posterior acromioclavicular joint was digitized. Sensor data was transformed using Euler sequences to clinically relevant angles of the humerus relative to the scapula and the humerus and the scapula relative to the thorax. Data were collected at $100 \mathrm{~Hz}$.

\section{Statistical Analysis}

Rank order (lowest to highest) of the mechanical impingement risk score for each CRT exercise is a novel technique that captures risk for CRT exercises at specific humerothoracic elevation angles associated with subacromial and/or internal mechanical impingement. Each score is calculated from two components: (1) the magnitude of kinematic combinations (for each scapular and glenohumeral variable) and (2) exposure, or time spent in "impingement risk ranges." The two components are described first, followed by the process for combining them to obtain the overall mechanical impingement risk score.

\section{Magnitude of Kinematic Combinations Component}

The continuous scapula and glenohumeral kinematic data collected during the concentric phase of each CRT exercise for each subject were compared with previously published data in nondisabled individuals at comparable angles of humerothoracic elevation (Table 2) [31]. Decreased scapular posterior tilt and upward rotation and increased scapular and glenohumeral internal rotation were considered rotations of detrimental kinematics. Data between $45^{\circ}$ and $60^{\circ}$ (subacromial) of humerothoracic elevation were analyzed for each exercise. A value between one and three was assigned (with three being most kine- matically detrimental) based on the number of standard deviations each kinematic data point differs from comparison means (Figure 4). A point value of one was assigned for measurements that were less than one standard deviation away from the comparison mean (detrimental direction or any amount in the favorable direction). A point value of two was assigned if the measurement was between one and two standard deviations (detrimental direction) from the comparison mean. A point value of three was assigned if the measurement was greater than two standard deviations (detrimental direction) from the comparison mean. A point value of zero was assigned if the humerus did not pass through $45^{\circ}$ to $60^{\circ}$ of humerothoracic elevation during the exercise. The resulting values for each of the scapular and glenohumeral kinematics were combined into the kinematic component of the mechanical risk score. This same process was repeated between $105^{\circ}$ and $120^{\circ}$ (internal) of humerothoracic elevation for each exercise.

\section{Exposure Component}

The total time spent in an impingement risk range during each CRT exercise, normalized to the concentric phase of each exercise, was also factored into the mechanical impingement risk score. For example, if the concentric phase of a particular exercise spanned $3 \mathrm{~s}$, and $2 \mathrm{~s}$ were within a subacromial or internal impingement range, then the exposure component would be 67 percent.

\section{Mechanical Impingement Risk Score Calculation (Combination of Magnitude of Kinematic Combinations and Exposure Components)}

The mechanical risk scores were obtained by integrating the magnitude of kinematic deviation from comparison data for each frame with respect to the normalized time (exposure within impingement ranges). The resulting mechanical impingement risk scores were averaged across all 10 repetitions of each exercise. A higher score would represent a greater mechanical impingement risk.

Table 2.

Comparison data for humerothoracic elevation in scapular plane. Kinematic data (mean \pm standard deviation) based on right-handed coordinate system provided at various degrees humerothoracic elevation using bone-fixed tracking method [31].

\begin{tabular}{lccrr}
\hline \multirow{2}{*}{ Kinematic Variable } & \multicolumn{3}{c}{ Humerothoracic Elevation Angle } \\
\cline { 2 - 5 } & $\mathbf{4 5}^{\circ}$ & $\mathbf{6 0}^{\circ}$ & $\mathbf{1 0 5}^{\circ}$ & $39.1 \pm 10.2$ \\
\hline Scapulothoracic Internal Rotation (+) & $38.7 \pm 9.9$ & $38.8 \pm 10.1$ & $37.2 \pm 10.3$ & $-43.5 \pm 7.5$ \\
Scapulothoracic Upward Rotation (-) & $-20.9 \pm 6.8$ & $-26.0 \pm 7.2$ & $-38.6 \pm 7.5$ & $2.7 \pm 5.4$ \\
Scapulothoracic Posterior Tilting (+) & $-9.0 \pm 4.8$ & $-6.8 \pm 4.6$ & $-0.7 \pm 4.8$ & $-62.0 \pm 8.6$ \\
Glenohumeral External Rotation (-) & $-54.7 \pm 12.4$ & $-57.0 \pm 11.6$ & $-61.0 \pm 8.6$ & $\mathbf{1 2 0}^{\circ}$ \\
\hline \hline
\end{tabular}




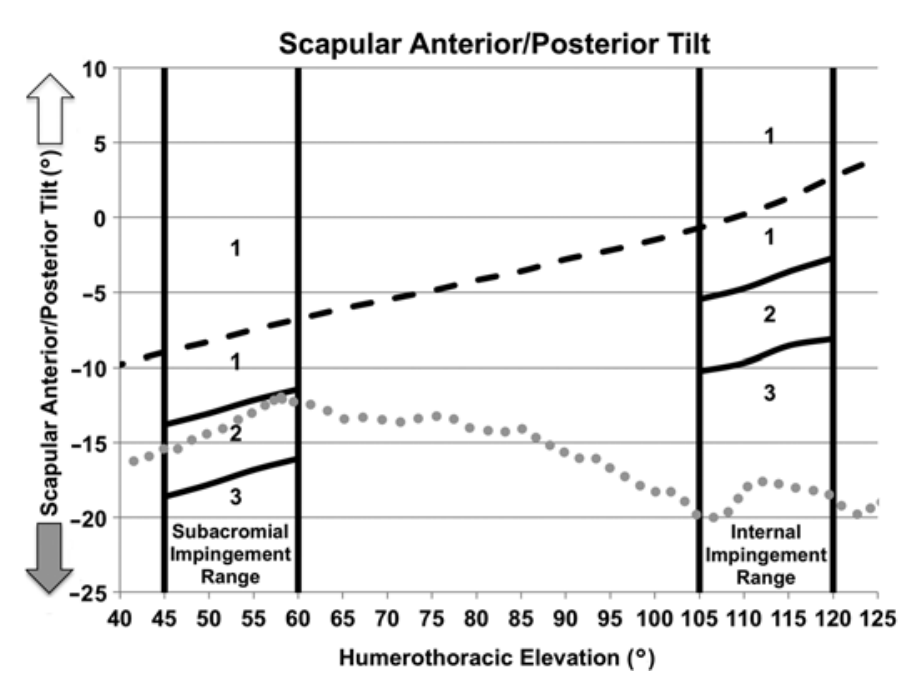

Figure 4.

Magnitude of kinematic combinations component. Comparison data depicted by dashed black line. Representative subject data for scapular anterior tilt during lat pulldown depicted by dotted line. Favorable kinematic direction (posterior tilt) indicated by white arrow. Detrimental kinematic direction (anterior tilt) indicated by gray arrow. Point value of $1=$ less than one standard deviation away from comparison mean (detrimental direction or any amount in the favorable direction), 2 = between one and two standard deviations (detrimental direction) from comparison mean, 3 = greater than two standard deviations (detrimental direction) from comparison mean.

For exercises typically performed at lower levels of humeral elevation, such as chest press, seated row, and rickshaw, the maximum possible subacromial mechanical impingement risk score would be 12 points (kinematic score of 3 for all four kinematic variables at 100\% exposure). For exercises that spanned a larger range of humeral elevation, such as the lat pulldown and the overhead press, the maximum possible subacromial or internal impingement scores would also be 12 points. However, scores will be less than this maximum if a portion of the exercise is performed at lower (subacromial) and another portion at upper (internal) ranges of humerothoracic elevation since the exposure time would be reduced at each of the impingement ranges.

\section{RESULTS}

All five exercises were performed at lower ranges of humerothoracic elevation and included in the subacromial mechanical impingement risk analysis. Two of the exercises (overhead press and lat pulldown) also spanned upper ranges of humerothoracic elevation and were included in the internal mechanical impingement risk analysis. All four kinematic variables contributed to the magnitude component of the mechanical impingement risk score, ranging individually from 0.9 to 2.4 (subacromial) and 1.3 to 2.9 (internal; Table 3 ) (out of a maximum of 3). During CRT, exposure also occurred in the impingement ranges spanning from 10.9 to 42.5 percent (subacromial) and 14.3 to 17.5 percent (internal) and contributed to the mechanical impingement risk score (Table 3). Table 3 shows the mechanical impingement risk scores, reflecting the combined kinematic and exposure contribution.

The mean mechanical impingement risk scores at $45^{\circ}$ to $60^{\circ}$ humerothoracic elevation resulted in a rank order for lowest-to-highest subacromial mechanical impingement risk: overhead press $(0.6 \pm 0.5$ points or lowest risk), lat pulldown (1.2 \pm 0.5 points), chest press ( $2.4 \pm$ 2.8 points), seated row ( $2.7 \pm 1.6$ points), and rickshaw (3.4 \pm 2.3 points or highest risk) (Figure 5). The mean mechanical impingement risk scores at $105^{\circ}$ to $120^{\circ}$ humerothoracic elevation resulted in a rank order for lowest-to-highest internal mechanical impingement risk: lat pulldown (1.2 \pm 0.5 points or lowest risk) and overhead press (1.3 \pm 0.5 points or highest risk) (Figure 5).

\section{DISCUSSION}

This study provides the first evaluation of shoulder kinematics during the execution of wheelchair-based UL CRT exercises that may help to guide shoulder-healthy CRT recommendations for individuals with paraplegia. The outcomes from this study indicate that certain CRT exercises are completed in ranges of humerothoracic elevations that may place the shoulder at increased risk for mechanical impingement. The findings provide a better understanding of the potential mechanical impingement risk associated with various CRT exercises that are frequently recommended for individuals with SCI.

Without complex three-dimensional modeling techniques, it is difficult to obtain a comprehensive understanding of mechanical impingement risk when analyzing scapular and glenohumeral kinematics as isolated variables. One direction of rotation may be in a favorable direction while the other(s) may be in a detrimental direction. The technique used in this study provides an innovative way to 
combine the magnitude of scapular and glenohumeral motion that occurs at the shoulder joint, as well as the exposure or percentage of time spent in the subacromial and/or internal impingement ranges, thus generating a mechanical impingement risk score.
Additionally, exposure or frequency has been previously considered in other SCI studies as a contributor to increased risk for shoulder pain. For example, wheelchair propulsion has a peak glenohumeral joint reaction force of only $304 \mathrm{~N}$ for individuals with paraplegia as compared

Table 3.

Mechanical impingement risk score. Magnitude component: average deviation \pm standard deviation from comparison data for all four kinematic variables of interest during circuit resistance training (CRT) exercises. Maximum score for each kinematic variable is 3 points. Exposure component: average deviation \pm standard deviation reflecting percentage of time in mechanical impingement risk ranges during CRT exercises. Higher values indicate greater mechanical impingement risk.

\begin{tabular}{|c|c|c|c|c|c|c|c|}
\hline \multirow{2}{*}{ Exercise } & \multicolumn{4}{|c|}{ Magnitude } & \multirow{2}{*}{ Exposure (\%) } & \multirow{2}{*}{$\begin{array}{l}\text { Mechanical } \\
\text { Impingement } \\
\text { Risk Score }\end{array}$} & \multirow{2}{*}{ Risk Range } \\
\hline & Scapular AT & Scapular IR & Scapular DR & GHIR & & & \\
\hline$\overline{\text { Row }}$ & $2.00 \pm 0.87$ & $1.40 \pm 0.64$ & $1.50 \pm 0.76$ & $1.40 \pm 0.68$ & $41.2 \pm 21.8$ & $2.7 \pm 1.6$ & Subacromial \\
\hline Chest Press & $1.40 \pm 1.22$ & $1.00 \pm 0.82$ & $1.10 \pm 1.09$ & $1.30 \pm 1.17$ & $30.2 \pm 32.2$ & $2.4 \pm 2.8$ & Subacromial \\
\hline Lat Pulldown & $1.90 \pm 0.90$ & $1.40 \pm 0.60$ & $1.40 \pm 0.66$ & $1.10 \pm 0.34$ & $20.9 \pm 8.5$ & $1.2 \pm 0.5$ & Subacromial \\
\hline Overhead Press & $2.20 \pm 0.84$ & $1.70 \pm 0.71$ & $2.00 \pm 0.81$ & $1.60 \pm 0.82$ & $17.5 \pm 3.9$ & $1.3 \pm 0.5$ & Internal \\
\hline
\end{tabular}

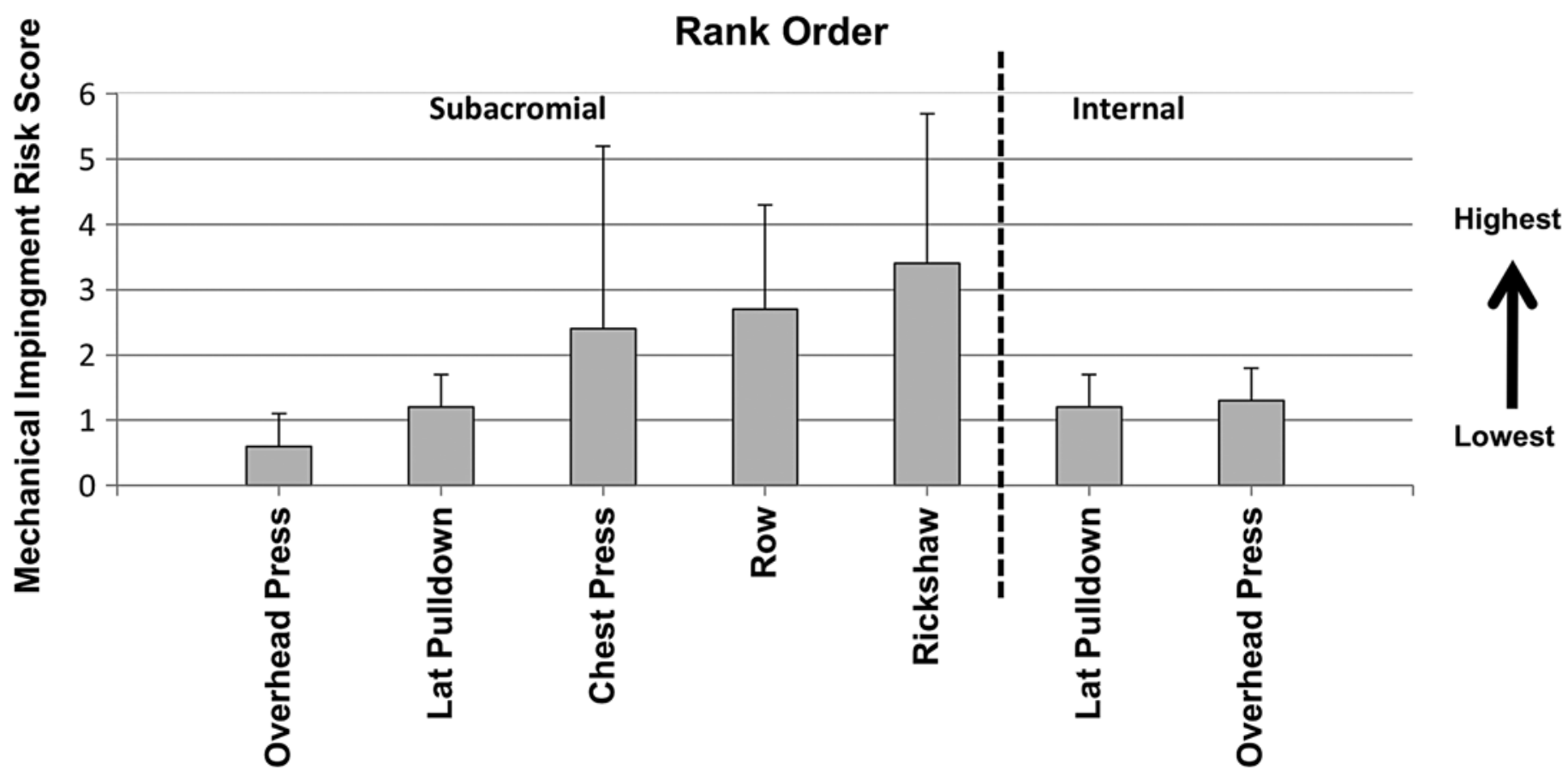

\section{Exercise}

Figure 5.

Rank order of circuit resistance training (CRT) exercises by Mechanical Impingement Risk Score (mean \pm standard deviation). Five CRT exercises are performed at lower humerothoracic elevation angles representing subacromial mechanical impingement risk and two CRT exercises are performed at upper humerothoracic elevation angles representing internal mechanical impingement risk. 
with a weight relief lift with a peak of 1,248.1 $\mathrm{N}$ [42]. Although the forces during wheelchair propulsion were smaller than the weight relief lift, the exposure or time spent performing wheelchair propulsion (1,800 propulsions/day) [43] may equalize the shoulder risk. As in this study, exposure or time spent performing a task must be factored into mechanical impingement risk alongside the magnitude of the kinematics.

From the rank-order analysis, the rickshaw (3.4 \pm 2.3 points) had the highest subacromial risk, whereas the overhead press (1.3 \pm 0.5 points) and lat pulldown (1.2 \pm 0.5 points) had the highest internal mechanical impingement risk (Figure 5). Of all CRT exercises, the rickshaw was highlighted as the exercise of most concern because it had the greatest combination of both magnitude and exposure corresponding with increased subacromial mechanical impingement risk (Figure 5). Of note, almost half $(42.5 \%)$ of the exercise was performed in a subacromial impingement range. In a prior telephone survey (conducted by L.R.) to 14 SCI Model System Centers, 71 percent of the reporting centers used the rickshaw as part of their program and 86 percent used some variation of a multistation gym. These findings suggest that the rickshaw is commonly prescribed but should be recommended with caution when required to achieve specific goals of functional independence. Upon further review of the individual data, trends were consistent among the individual participants. Two participants performed the rickshaw without entering the subacromial impingement range $\left(45-60^{\circ}\right)$. Because these two participants are still included in the analysis, this contributed to a larger standard deviation and a lower mean for the rickshaw. Yet the rickshaw still had the highest subacromial impingement risk score. For example, the rickshaw ranked first when looking at the percentage of individuals who had this exercise as their highest subacromial impingement risk score (39\%). This was followed by chest press (33\%), seated row (22\%), and lat pulldown (6\%).

Although the lat pulldown and the overhead press do spend a portion of the exercise in both a subacromial risk range and an internal impingement risk range, the exposure component is relatively low (20.9\% and $10.9 \%$, respectively) and thus the overall mechanical impingement risk scores remain low (Figure 5). For example, the highest mechanical impingement risk scores ever achieved by the lat pulldown (1.2 \pm 0.5 points) and the overhead press (1.3 \pm 0.5 points) are less than half that of the rickshaw ( $3.4 \pm 2.3$ points). At lower range humerothoracic elevation, the chest press and the seated row should be recommended with more caution if subacromial impingement symptoms exist. Although these four exercises exhibit smaller magnitudes of kinematic deviation from comparison data as compared with the rickshaw, all have relatively high exposure to impingement ranges (up to $41 \%$ subacromial and $17.5 \%$ internal) (Table 3).

In addition to overall goals of increased strength or function, exercise recommendations must take into account patient-specific considerations, including the presence of existing pain (subacromial or internal). Individuals with complaints of subacromial impingement should be encouraged to avoid the rickshaw, but may want to cautiously consider the other CRT exercises as well (chest press, seated row, lat pulldown, and overhead press). Individuals with complaints of internal impingement should consider avoiding higher ranges of elevation during both the lat pulldown and overhead press. Alternatively, participants may want to reduce the number of repetitions or frequency of the activity. Each exercise should be thoughtfully prescribed as part of a customized program to meet specific functional goals as needed. The functional benefits obtained from each exercise must be weighed alongside the risk of shoulder mechanical impingement. The secondary benefits of exercise are undeniable in the SCI population. To gain these health benefits while minimizing shoulder mechanical impingement risk, it is important to add variety to the workout program. For example, a varied exercise program of CRT, a home stretching and strengthening program emphasizing the rotator cuff musculature [35], yoga, aquatic therapy, cardiovascular exercise, and/or core strengthening may help achieve health benefits while minimizing overuse injuries. Specifically, strengthening of the rotator cuff has the potential to reduce mechanical impingement and thus should be considered not only as a crucial part of a home exercise program but also in the early phases of rehabilitation following SCI. Because of the critical role, particularly with regard to preventing excess superior translation of humerus, and in producing humeral external rotation, further research should explore the importance of rotator cuff strengthening in a comprehensive rehabilitation program that minimizes shoulder mechanical impingement risk.

Future studies should also consider the effect of sitting posture and balance on biomechanics during CRT. Improved wheelchair posture and/or modifications to the CRT equipment that provide external trunk stabilization may prove to minimize shoulder mechanical impingement risk. Previous studies in the nondisabled population have found a link between trunk posture and shoulder kinematics, including increased scapular anterior tilt and elevation [44]. 
Note that this current study did not attempt to "fix" postural deviations; rather, subjects were evaluated using the CRT equipment as they would in a "real-life" exercise program. Clinically, it is common for individuals with SCI to assume a "slouched" or "C" position consisting of posterior pelvic tilt, reduced lumbar lordosis, and increased thoracic kyphosis. A slouched position may be attributed to absent or impaired innervation of key postural muscles. This posture provides the wheelchair user with improved short sitting balance, especially during UL activities including CRT (Figure 6). Individuals with SCI demonstrating a slouched rest posture are likely to have altered and potentially detrimental shoulder kinematic patterns before they even begin CRT. Additionally, various hand position options for the same exercise should be investigated to determine whether any biomechanical advantage exists.

As noted earlier in the rank-order analysis, from $45^{\circ}$ to $60^{\circ}$ humerothoracic elevation, exercises were rankordered from lowest to highest mechanical impingement risk (overhead press, lat pulldown, chest press, seated row, and rickshaw). The stability offered or the posture assumed during these CRT exercises may have positively or negatively affected the kinematic patterns observed at the glenohumeral joint. For example, subjects were supported posteriorly by their backrest during the overhead press, lat pulldown, and chest press, which were rankordered more favorably with regard to subacromial impingement than either the seated row or rickshaw. During the seated row, some subjects flexed their head and trunk over the "stabilizing" chest pad in order to increase stability (Figure 6). Additionally, subjects often reported

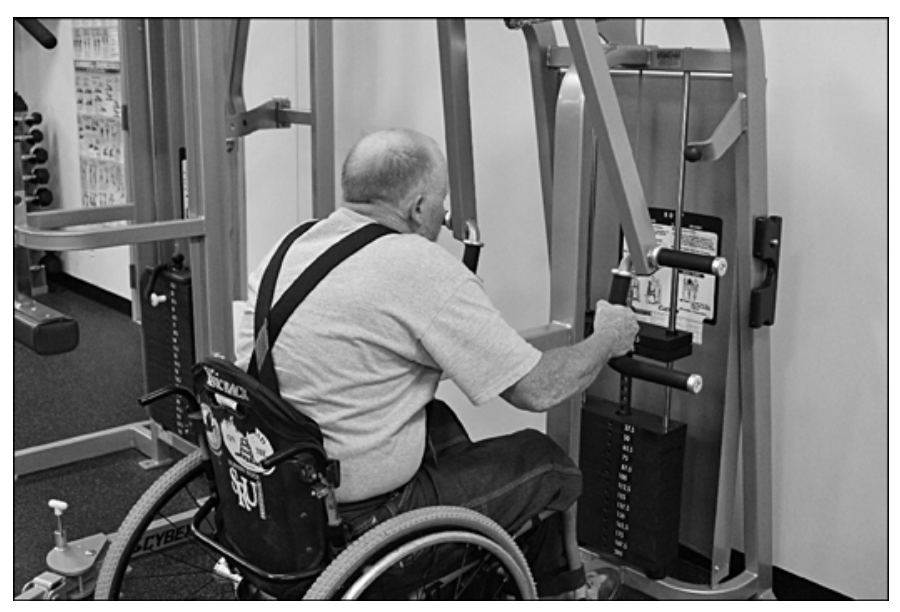

Figure 6.

Seated row. Subject performing seated row in "C" posture. difficulty maintaining their balance during the rickshaw and generally relied on one of two postural adaptations to maintain their balance: increased posterior pelvic tilt or increased trunk flexion. These postural adaptations may have contributed to the potentially detrimental shoulder kinematics observed during the seated row and rickshaw.

\section{LIMITATIONS}

The framework of this study was based on the assumption that "detrimental" scapulothoracic and glenohumeral kinematics affect either the subacromial space and/or internal structures and contribute to impingement symptoms. The analyses used in this project are the most current in vivo approaches and will need to be verified in the future with three-dimensional modeling. Skin motion artifact can occur with surface markers, especially over $120^{\circ}$ of humerothoracic elevation, which were approached during the overhead press and the lat pulldown exercises. However, the analysis was limited to less than $120^{\circ}$, reducing the magnitude of skin motion artifact. Participants also remained in their custom wheelchairs during CRT. Despite varying humerothoracic elevation angles between subjects, the variable wheelchair heights allowed data collection in a real-life setting.

Although Cybex Total Access equipment is appealing since wheelchair users do not have to transfer from their wheelchairs and it is available in real-life settings, it eliminates the adjustable seat-height feature offered to nondisabled individuals. Seat-height adjustability is an essential feature because it allows CRT to be performed at shoulder height and thus out of shoulder impingement ranges. Although an overhead adjustable lever can alter the lat pulldown height, other exercises (chest press, seated row, rickshaw and overhead press) must be performed at seat-heights dictated by the wheelchair. Because the seat height cannot be adjusted on this model, these exercises are frequently performed in impingement ranges. Future investigations should include exercise equipment with movable platforms capable of adjusting the height of the wheelchair relative to the machine or arm attachments that allow exercises to be performed at humerothoracic elevation angles outside of impingement ranges. Although it was beyond the scope of this investigation, future studies should expand beyond CRT to include biomechanical analysis of home exercise programs. Home exercise programs are also readily available and have been shown to significantly reduce shoulder pain in the SCI population [35]. 
The rank-order analysis relies on currently available comparison data obtained from a nondisabled group (Table 2). A comparison population of individuals with paraplegia and without shoulder pain who use a manual wheelchair for their primary means of locomotion might be considered ideal. However, a comparison with painfree, nondisabled individuals may actually highlight the increased risk for individuals with paraplegia. We have based the comparison on what are presumed optimal healthy kinematic values for given ranges of humeral elevation. If individuals with paraplegia assume a poor resting posture, then the resulting detrimental kinematics may be exacerbated when performing CRT exercises. Ultimately, the existing comparison data are applied exactly the same for each CRT exercise in the rank-order analysis. This negates any bias of the effect of this comparison on any one exercise over another.

The comparison data are also obtained during humerothoracic elevation in the scapular plane (Table 2). Humeral motion during CRT does not necessarily fall strictly within the scapular plane. However, the scapular plane is most representative of functional activities and captures many of the motions experienced during CRT as well as any single plane comparison can. Additionally, the technique selected for this study allowed our scapular and glenohumeral data to be compared with values at similar humerothoracic elevation angles.

\section{CONCLUSIONS}

Impingement risk scores that reflected both the magnitude of kinematic deviations and exposure provided a means to capture mechanical impingement risk associated with CRT. The rickshaw had the highest subacromial mechanical risk, whereas the overhead press and lat pulldown had the highest internal mechanical impingement risk. The rickshaw was highlighted as the most concerning exercise because it had the greatest combination of magnitude and exposure corresponding with increased subacromial mechanical impingement risk.

\section{ACKNOWLEDGMENTS}

\section{Author Contributions:}

Study concept and design: L. M. Riek, P. M. Ludewig,

D. A. Nawoczenski.

Acquisition of data: L. M. Riek.
Analysis and interpretation of data: L. M. Riek, P. M. Ludewig, D. A. Nawoczenski.

Drafting of manuscript: L. M. Riek.

Critical revision of manuscript for important intellectual content:

L. M. Riek, P. M. Ludewig, D. A. Nawoczenski.

Financial Disclosures: The authors have declared that no competing interests exist.

Funding/Support: This study was unfunded at the time of manuscript preparation.

Additional Contributions: The authors acknowledge Joshua Tome and Jonathan Riek for their assistance with this project.

Institutional Review: All investigation involving human subjects and the use of patient data for research purposes was approved by the committee on research ethics at the University of Rochester in accordance with the Declaration of the World Medical Association. Prior to participation, all participants signed institution-approved human subject informed consent documents.

Participant Follow-up: The authors have no plans to notify all the study subjects of the publication of this article because of a lack of contact information. For those participants seen on a regular basis, the authors will encourage them to check the Journal of Rehabilitation Research and Development Web site.

Disclosures: A portion of this manuscript was presented at the American Physical Therapy Association Combined Sections Meeting annual conference in Chicago, Illinois, in February 2012.

\section{REFERENCES}

1. Gupta N, White KT, Sandford PR. Body mass index in spinal cord injury - a retrospective study. Spinal Cord. 2006; 44(2):92-94. [PMID:16030513] http://dx.doi.org/10.1038/sj.sc.3101790

2. Weaver FM, Collins EG, Kurichi J, Miskevics S, Smith B, Rajan S, Gater D. Prevalence of obesity and high blood pressure in veterans with spinal cord injuries and disorders: a retrospective review. Am J Phys Med Rehabil. 2007; 86(1):22-29. [PMID:17304685] http://dx.doi.org/10.1097/PHM.0b013e31802b8937

3. Jacobs PL, Nash MS. Exercise recommendations for individuals with spinal cord injury. Sports Med. 2004;34(11): 727-51. [PMID:15456347] http://dx.doi.org/10.2165/00007256-200434110-00003

4. Lavela SL, Weaver FM, Goldstein B, Chen K, Miskevics S, Rajan S, Gater DR Jr. Diabetes mellitus in individuals with spinal cord injury or disorder. J Spinal Cord Med. 2006; 29(4):387-95. [PMID:17044389]

5. Nash MS, van de Ven I, van Elk N, Johnson BM. Effects of circuit resistance training on fitness attributes and upperextremity pain in middle-aged men with paraplegia. Arch Phys Med Rehabil. 2007;88(1):70-75. [PMID:17207678] http://dx.doi.org/10.1016/j.apmr.2006.10.003

6. Hicks AL, Martin KA, Ditor DS, Latimer AE, Craven C, Bugaresti J, McCartney N. Long-term exercise training in 
persons with spinal cord injury: effects on strength, arm ergometry performance and psychological well-being. Spinal Cord. 2003;41(1):34-43. [PMID:12494319]

http://dx.doi.org/10.1038/sj.sc.3101389

7. Jacobs PL, Nash MS, Rusinowski JW. Circuit training provides cardiorespiratory and strength benefits in persons with paraplegia. Med Sci Sports Exerc. 2001;33(5):711-17. [PMID:11323537] http://dx.doi.org/10.1097/00005768-200105000-00005

8. Nash MS, Jacobs PL, Mendez AJ, Goldberg RB. Circuit resistance training improves the atherogenic lipid profiles of persons with chronic paraplegia. J Spinal Cord Med. 2001;24(1):2-9. [PMID:11587430]

9. Jacobs PL. Effects of resistance and endurance training in persons with paraplegia. Med Sci Sports Exerc. 2009; 41(5):992-97. [PMID:19346989] http://dx.doi.org/10.1249/MSS.0b013e318191757f

10. Curtis KA, Drysdale GA, Lanza RD, Kolber M, Vitolo RS, West R. Shoulder pain in wheelchair users with tetraplegia and paraplegia. Arch Phys Med Rehabil. 1999;80(4):453-57. [PMID:10206610] http://dx.doi.org/10.1016/S0003-9993(99)90285-X

11. Gironda RJ, Clark ME, Neugaard B, Nelson A. Upper limb pain in a national sample of veterans with paraplegia. J Spinal Cord Med. 2004;27(2):120-27. [PMID:15162882]

12. Alm M, Saraste H, Norrbrink C. Shoulder pain in persons with thoracic spinal cord injury: prevalence and characteristics. J Rehabil Med. 2008;40(4):277-83.

[PMID:18382823]

http://dx.doi.org/10.2340/16501977-0173

13. McCasland LD, Budiman-Mak E, Weaver FM, Adams E, Miskevics S. Shoulder pain in the traumatically injured spinal cord patient: evaluation of risk factors and function. J Clin Rheumatol. 2006;12(4):179-86. [PMID:16891921] http://dx.doi.org/10.1097/01.rhu.0000230532.54403.25

14. Bayley JC, Cochran TP, Sledge CB. The weight-bearing shoulder. The impingement syndrome in paraplegics. J Bone Joint Surg Am. 1987;69(5):676-78. [PMID:3597466]

15. Samuelsson KA, Tropp H, Gerdle B. Shoulder pain and its consequences in paraplegic spinal cord-injured, wheelchair users. Spinal Cord. 2004;42(1):41-46. [PMID:14713943] http://dx.doi.org/10.1038/sj.sc.3101490

16. Silfverskiold J, Waters RL. Shoulder pain and functional disability in spinal cord injury patients. Clin Orthop Relat Res. 1991;272(272):141-45. [PMID:1934724]

17. Neer CS 2nd. Anterior acromioplasty for the chronic impingement syndrome in the shoulder: a preliminary report. J Bone Joint Surg Am. 1972;54(1):41-50. [PMID:5054450]

18. Neer CS 2nd. Impingement lesions. Clin Orthop Relat Res. 1983;173(173):70-77. [PMID:6825348]
19. Campbell RS, Dunn A. External impingement of the shoulder. Semin Musculoskelet Radiol. 2008;12(2):107-26. [PMID:18509791] http://dx.doi.org/10.1055/s-2008-1078699

20. Grainger AJ. Internal impingement syndromes of the shoulder. Semin Musculoskelet Radiol. 2008;12(2):127-35. [PMID:18509792] http://dx.doi.org/10.1055/s-2008-1078700

21. Heyworth BE, Williams RJ 3rd. Internal impingement of the shoulder. Am J Sports Med. 2009;37(5):1024-37. [PMID:19059895] http://dx.doi.org/10.1177/0363546508324966

22. Ludewig PM, Reynolds JF. The association of scapular kinematics and glenohumeral joint pathologies. J Orthop Sports Phys Ther. 2009;39(2):90-104. [PMID:19194022]

23. Bey MJ, Brock SK, Beierwaltes WN, Zauel R, Kolowich PA, Lock TR. In vivo measurement of subacromial space width during shoulder elevation: technique and preliminary results in patients following unilateral rotator cuff repair. Clin Biomech (Bristol, Avon). 2007;22(7):767-73. [PMID:17560699] http://dx.doi.org/10.1016/j.clinbiomech.2007.04.006

24. Giphart JE, van der Meijden OA, Millett PJ. The effects of arm elevation on the 3-dimensional acromiohumeral distance: a biplane fluoroscopy study with normative data. J Shoulder Elbow Surg. 2012;21(11):1593-1600.

[PMID:22361718] http://dx.doi.org/10.1016/j.jse.2011.11.023

25. Bigliani LU, Levine WN. Current concepts review: Subacromial impingement syndrome. J Bone Joint Surg Am. 1997;79(12):1844-68.

26. Borstad JD, Ludewig PM. Comparison of scapular kinematics between elevation and lowering of the arm in the scapular plane. Clin Biomech (Bristol, Avon). 2002;17(9-10):650-59. [PMID:12446161] http://dx.doi.org/10.1016/S0268-0033(02)00136-5

27. Ludewig PM, Cook TM. Alterations in shoulder kinematics and associated muscle activity in people with symptoms of shoulder impingement. Phys Ther. 2000;80(3):276-91. [PMID:10696154]

28. Lin JJ, Hanten WP, Olson SL, Roddey TS, Soto-quijano DA, Lim HK, Sherwood AM. Shoulder dysfunction assessment: self-report and impaired scapular movements. Phys Ther. 2006;86(8):1065-74. [PMID:16879041]

29. Lin JJ, Hanten WP, Olson SL, Roddey TS, Soto-Quijano DA, Lim HK, Sherwood AM. Functional activity characteristics of individuals with shoulder dysfunctions. J Electromyogr Kinesiol. 2005;15(6):576-86. [PMID:16179197] http://dx.doi.org/10.1016/j.jelekin.2005.01.006

30. Hébert LJ, Moffet H, McFadyen BJ, Dionne CE. Scapular behavior in shoulder impingement syndrome. Arch Phys 
Med Rehabil. 2002;83(1):60-69. [PMID:11782834]

http://dx.doi.org/10.1053/apmr.2002.27471

31. Ludewig PM, Phadke V, Braman JP, Hassett DR, Cieminski CJ, LaPrade RF. Motion of the shoulder complex during multiplanar humeral elevation. J Bone Joint Surg Am. 2009;91(2):378-89. [PMID:19181982]

http://dx.doi.org/10.2106/JBJS.G.01483

32. Flatow EL, Soslowsky LJ, Ticker JB, Pawluk RJ, Hepler M, Ark J, Mow VC, Bigliani LU. Excursion of the rotator cuff under the acromion. Patterns of subacromial contact. Am J Sports Med. 1994;22(6):779-88. [PMID:7856802] http://dx.doi.org/10.1177/036354659402200609

33. Yanai T, Fuss FK, Fukunaga T. In vivo measurements of subacromial impingement: substantial compression develops in abduction with large internal rotation. Clin Biomech (Bristol, Avon). 2006;21(7):692-700. [PMID:16632128] http://dx.doi.org/10.1016/j.clinbiomech.2006.03.001

34. Nawoczenski DA, Ritter-Soronen JM, Wilson CM, Howe BA, Ludewig PM. Clinical trial of exercise for shoulder pain in chronic spinal injury. Phys Ther. 2006;86(12): 1604-18. [PMID:17138842]

http://dx.doi.org/10.2522/ptj.20060001

35. Mulroy SJ, Thompson L, Kemp B, Hatchett PP, Newsam CJ, Lupold DG, Haubert LL, Eberly V, Ge TT, Azen SP, Winstein CJ, Gordon J; Physical Therapy Clinical Research Network (PTClinResNet). Strengthening and optimal movements for painful shoulders (STOMPS) in chronic spinal cord injury: a randomized controlled trial. Phys Ther. 2011;91(3):305-24. [PMID:21292803] http://dx.doi.org/10.2522/ptj.20100182

36. Riek LM, Ludewig PM, Nawoczenski DA. Comparative shoulder kinematics during free standing, standing depression lifts and daily functional activities in persons with paraplegia: considerations for shoulder health. Spinal Cord. 2008;46(5):335-43. [PMID:18026174] http://dx.doi.org/10.1038/sj.sc.3102140

37. Curtis KA, Roach KE, Applegate EB, Amar T, Benbow CS, Genecco TD, Gualano J. Development of the Wheelchair User's Shoulder Pain Index (WUSPI). Paraplegia. 1995;33(5):290-93. [PMID:7630657] http://dx.doi.org/10.1038/sc.1995.65

38. Nash MS, Jacobs PL, Woods JM, Clark JE, Pray TA, Pumarejo AE. A comparison of 2 circuit exercise training techniques for eliciting matched metabolic responses in persons with paraplegia. Arch Phys Med Rehabil. 2002; 83(2):201-9. [PMID:11833023] http://dx.doi.org/10.1053/apmr.2002.28011

39. Nawoczenski DA, Clobes SM, Gore SL, Neu JL, Olsen JE, Borstad JD, Ludewig PM. Three-dimensional shoulder kinematics during a pressure relief technique and wheelchair transfer. Arch Phys Med Rehabil. 2003;84(9):1293-1300.

\section{[PMID:13680564]}

http://dx.doi.org/10.1016/S0003-9993(03)00260-0

40. Karduna AR, McClure PW, Michener LA, Sennett B. Dynamic measurements of three-dimensional scapular kinematics: a validation study. J Biomech Eng. 2001;123(2): 184-90. [PMID:11340880] http://dx.doi.org/10.1115/1.1351892

41. Wu G, van der Helm FC, Veeger HE, Makhsous M, Van Roy P, Anglin C, Nagels J, Karduna AR, McQuade K, Wang X, Werner FW, Buchholz B; International Society of Biomechanics. ISB recommendation on definitions of joint coordinate systems of various joints for the reporting of human joint motion-Part II: shoulder, elbow, wrist and hand. J Biomech. 2005;38(5):981-92. [PMID:15844264] http://dx.doi.org/10.1016/j.jbiomech.2004.05.042

42. van Drongelen S, van der Woude LH, Veeger HE. Load on the shoulder complex during wheelchair propulsion and weight relief lifting. Clin Biomech (Bristol, Avon). 2011; 26(5):452-57. [PMID:21316822]

http://dx.doi.org/10.1016/j.clinbiomech.2011.01.006

43. van der Woude L, de Groot S, van Drongelen S, Janssen T, Haisma JA, Valent LJ, Veeger D. Evaluation of manual wheelchair performance in everyday life. Top Spinal Cord Inj Rehabil. 2009;15(2):1-15.

http://dx.doi.org/10.1310/sci1502-1

44. Kebaetse M, McClure P, Pratt NA. Thoracic position effect on shoulder range of motion, strength, and three-dimensional scapular kinematics. Arch Phys Med Rehabil. 1999;80(8): 945-50. [PMID:10453773] http://dx.doi.org/10.1016/S0003-9993(99)90088-6

Submitted for publication June 12, 2012. Accepted in revised form December 18, 2012.

This article and any supplementary material should be cited as follows:

Riek LM, Ludewig PM, Nawoczenski DA. How "healthy" is circuit resistance training following paraplegia? A kinematic analysis associated with shoulder mechanical impingement risk. J Rehabil Res Dev. 2013; 50(6):861-74. http://dx.doi.org/10.1682/JRRD.2012.06.0112

ResearcherID/ORCID: Linda M. Riek, DPT, PhD: G5302-2013; Paula M. Ludewig, PT, PhD: G-5418-2013; Deborah A. Nawoczenski, PT, PhD: G-5340-2013

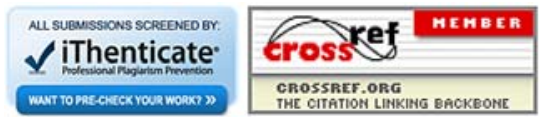


